\title{
Pourquoi une revue scientifique internationale portant sur l'intégration des TIC en pédagogie universitaire?
}

\section{Thierry Karsenti, rédacteur en chef}

Université de Montréal (Canada)

revue-redac@crepuq.qc.ca

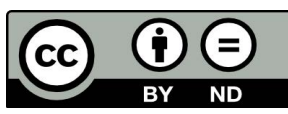

(C)Auteur(s). Cette œuvre, disponible à http://ritpu.ca/IMG/pdf/ritpu0101_karsenti_FR-2.pdf, est mise à disposition selon les termes de la licence Creative Commons Attribution Pas de Modification 2.5 Canada :

Un jour, les gens apprendront à travers des circuits électroniques. Marshall McLuhan, 1965

http://creativecommons.org/licences/by-nd/2.5/ca/deed.fr

$\mathrm{Q}$ uelques années après avoir réalisé un film destiné à l'éducation en 1911, Thomas Edison a dit: "Les livres seront incessamment désuets. Les étudiants apprendront bientôt à travers leurs yeux. Il est possible d'enseigner toutes les facettes du savoir humain par le film. Notre système d'éducation sera complètement transformé d'ici dix ans " '. Depuis la promesse d'Edison de révolutionner l'enseignement par le film, les expériences d'intégration des technologies en pédagogie universitaire ont connu plusieurs développements : l'utilisation du téléphone, de la radio, de la télévision, de la vidéo, de l'ordinateur, ainsi que des technologies de l'information et de la communication (TIC).

Toutefois, depuis le début des années 1980, où, pour la seule fois de son histoire, Time Magazine reconnaissait l'importance de l'ordinateur en octroyant la palme de "personnalité » de l'année à une machine, c'est surtout l'ordinateur et Internet qui connaissent une popularité grandissante en pédagogie universitaire. Dans l'introduction du numéro, le rédacteur en chef de Time Magazine, John A. Meyers, écrivait: «Plusieurs candidats humains auraient pu représenter 1982, mais aucun ne symbolisera autant l'année qui vient de passer, ni ne sera considéré dans l'histoire comme aussi marquant qu'une machine: l'ordinateur ». De nos jours, les TIC, susceptibles de contribuer à la démocratisation de l'accès au savoir, sont d'une puissance et d'une convivialité que ne pouvait imaginer Edison. Elles combinent à la fois les textes, le son, l'image, l'interactivité, la programmation; elles peuvent " enregistrer » et retransmettre aux quatre coins du globe. La société du savoir « promise dans les années 1970, vantée dans les années 1980 et envisagée dans les années 1990 avec un respect mêlé de crainte et d'incrédulité » est devenue, au XXI ${ }^{e}$ siècle, une réalité incontournable (Gouvernement du Canada, 2000).

Cela fait déjà quelques années que la Conférence des recteurs et des principaux des universités du Québec (CREPUQ) souligne que l'université évolue dans un contexte de mutation du rapport au savoir et entre résolument dans l'univers de l'information numérique et d'Internet. Pour la CREPUQ (1999), cette révolution technologique procure des avantages que les universités peuvent et doivent mettre en valeur dans le respect de leur mission fondamentale de formation et de recherche. En effet, l'intégration et la mise à profit des diverses fonctionnalités des TIC permettraient d'élargir tout le domaine de la formation supérieure en facilitant les collaborations et en améliorant sensiblement les environnements de recherche et les conditions de production intellectuelle.
Au cours des années, la terminologie employée pour décrire l'arrimage entre l'ordinateur et la pédagogie universitaire a reflété l'évolution des finalités de l'enseignement. On parle d'abord d'EPO (Enseignement programmé par ordinateur), puis d'EAO (Enseignement assisté par ordinateur) et, éventuellement, d'APO (Applications pédagogiques de l'ordinateur). Anciennement ancrée dans une logique de diffusion du savoir, l'utilisation de l'ordinateur et des TIC en pédagogie universitaire doit maintenant affronter celle de la navigation du savoir, car, en plus de permettre l'accès à des ressources jusqu'alors difficilement accessibles, les TIC facilitent aussi une mutualisation des connaissances.

Sur le plan de la formation à distance (FAD), on constate aussi une évolution du discours : cours en ligne, cours "sur le Web" et, plus récemment, formations ouvertes et à distance (FOAD). Les FOAD reflètent d'ailleurs un virage pédagogique emprunté récemment par les formateurs à l'université. En effet, alors que l'individualisation de l'enseignement représentait le principal objectif des formations à distance, la pédagogie universitaire semble aujourd'hui plus préoccupée par l'apprentissage en réseau et la constitution de communautés d'apprentissage. 
Par delà le discours qui proclame leurs «vertus intrinsèques » sur le plan de l'apprentissage, l'arrimage entre les TIC et la pédagogie universitaire nous paraît un univers de recherche en soi qu'il semble nécessaire de documenter de façon scientifique. On retrouve d'abord à l'université une variété de contextes où sont intégrées des technologies dont l'efficacité réelle n'est pas analysée de façon systématique. Les expériences sont nombreuses et variées, qu'il s'agisse d'enseignement en présentiel soutenu par les TIC, de cours hybrides où sont combinés des rencontres à l'université et des modules de formation à distance ou encore de cours "strictement» à distance sans rencontre in situ entre les apprenants et le formateur. Malgré l'omniprésence des technologies dans les universités et la multiplication des expériences pilotes d'enseignement avec les TIC, non seulement plusieurs s'interrogent toujours sur leur efficacité réelle (voir Ruano-Borbalan, 2001; et Zhao et Frank, 2003), mais on retrouve encore aussi peu de recherches rigoureuses (voir Ungerleider, 2002) ou encore d'expériences minutieusement documentées.

Dans la foulée des travaux de Prof\&TIC de la Conférence des recteurs et des principaux des universités du Québec, il a été décidé de mettre en place la Revue internationale des technologies en pédagogie universitaire. La Revue est une initiative collective et innovatrice des universités du Québec. Son comité scientifique international regroupe des membres provenant d'Amérique du Nord, d'Amérique du Sud, de l'Europe et de l'Afrique. Elle a pour but la diffusion d'expériences et de pratiques pédagogiques, d'évaluations de formations ouvertes ou à distance, de réflexions critiques et de recherches portant sur l'intégration des technologies de l'information et de la communication en pédagogie universitaire. La Revue souhaite aussi exposer de multiples approches pédagogiques et technologiques, et présenter des expertises interdisciplinaires et des expériences universitaires différenciées.
La création de cette revue, c'est en premier lieu la réponse à trois grandes préoccupations formulées par un groupe composé de professeurs, de chercheurs et de formateurs qui œuvrent au sein d'universités du Québec. La Revue répond d'abord au besoin de diffusion scientifique d'expériences et de pratiques pédagogiques portant sur l'intégration des TIC en enseignement supérieur. Il semblait de plus en plus nécessaire, au Québec, mais aussi dans le reste de la francophonie, de mettre en place une revue scientifique internationale portant sur les technologies de l'information et de la communication en pédagogie universitaire, dont les textes sont soumis à une évaluation par un comité formé de pairs.

Cette revue répond également au besoin de valorisation de l'enseignement en contexte universitaire. Nombreux sont les professeurs, chargés de cours et autres formateurs qui hésitent à innover et à intégrer les TIC, non pas parce qu'ils n'y croient pas, mais plutôt parce que de tels efforts sont peu ou prou reconnus. En effet, dans leurs universités, ce sont habituellement les subventions, la recherche et les publications scientifiques qui prennent souvent le pas sur la valorisation de la pédagogie universitaire.

Enfin, la mise en place de cette revue scientifique se veut une manière de dépasser le discours simpliste sur l'intégration des TIC en pédagogie universitaire. Pour que tous les étudiants puissent, à terme, bénéficier d'une meilleure formation, les universités doivent faire appel aux multiples possibilités que leur offrent l'enseignement à distance et les nouvelles technologies. Cependant, si l'on veut dépasser le stade expérimental, il faut absolument ancrer et structurer les efforts d'intégration des TIC en fonction des résultats de la recherche scientifique. À la Revue internationale des technologies en pédagogie universitaire, on est donc bien décidé à pérenniser cet arrimage technopédagogique qui fait encore trop souvent figure d'exception et qui n'en est, pour certains, quà ses balbutiements.
Les nouvelles technologies de l'information et de la communication contribuentelles réellement à rehausser la formation universitaire et à démocratiser les savoirs fondamentaux? Qu'est-ce que les TIC permettent de faire sur le plan de la pédagogie universitaire? Dans la formation universitaire, les technologies deviennent-elles un passage obligé pour accéder aux savoirs ? Les TIC peuvent-elles améliorer les chances d'un plus grand nombre de personnes d'accéder à l'enseignement supérieur? Sont-elles réellement efficaces en pédagogie universitaire? Les formations ouvertes et à distance ontelles un véritable avenir? Autant de questions auxquelles la Revue internationale des technologies en pédagogie universitaire espère apporter des éléments de réponse par les textes qui y seront publiés.

\section{Bonne lecture!}

\section{Références}

CREPUQ (1999). Mise en valeur des technologies de l'information et des communications pour la formation universitaire. Montréal: Conférence des recteurs et des principaux des universités du Québec.

Gouvernement du Canada (2000). Un Canada branché. Ottawa: Presses du Gouvernement du Canada.

Ruano-Borbalan, J.-C. (2001). Risques et promesses de l'e-éducation. Sciences humaines. (32), 44-47.

Ungerleider, C. (2002). Information and Communication Technologies in Elementary and Secondary Education. Toronto: Conseil des ministres de l'Éducation du Canada.

Zhao, Y. \& Frank, K. A. (2003). Factors Affecting Technology Uses in Schools: An Ecological Perspective. American Educational Research Journal, 40(4), 807-840.

\section{Note}

1 Extraits d'un discours prononcé par Thomas Edison en 1913. Traduction libre. Source: National Digital Library, http://memory.loc.gov/ 